\title{
Hydrodynamic Analysis of Trimaran Vessels
}

\author{
M.R. Javanmardi, E. Jahanbakhsh, \\ M.S. Seif, H.Sayyaadi, \\ Sharif University of Technology
}

\begin{abstract}
Trimaran vessels are developed for different applications and hydrodynamic behavior of such vessels is different than usual mono-hulls. In this paper hydrodynamic resistance and maneuvering of a trimaran with Wigley body form are investigated. The effects of outriggers position in four different longitudinal and two transverse locations are studied. For hydrodynamic simulations a CFD code has been developed and used. This code is capable for simulating three dimensional, time dependent, two phases, viscous flow coupled with rigid body motion. Formulation and solution algorithm are described in detail. Different case studies have been performance and numerical results have shown good agreement with experimental data. Based on resistance and maneuvering simulation of the trimaran vessels different conclusion are made. The results show that positions of outriggers have great effect on resistance and maneuverability of trimaran. The present method can be further employed to investigate other hydrodynamic qualities of trimaran vessels.
\end{abstract}

Keywords: trimaran; resistance; maneuvering; computational fluid dynamic

\section{INTRODUCTION}

Wave making resistance is an important component of ship resistance. It is very effective at high speeds and will require more attention in designing of high speed ships. Normally large slenderness ratio is necessary to decrease the wave making resistance. Therefore the ship hull should be as slender as possible for attaining higher speeds. But the main drawback of the slenderness is that the transverse stability decreases. Hence to overcome this challenge, the single body must be changed to multi-hull with proper separation distance. It means that a trimaran vessel which is composed of a main slender body and two outriggers can be an appropriate solution to improve vessel transverse stability, while the efficient wave interaction, created by main body and outriggers is able to compensate for wetted surface increase and guaranties slender bodies with good stability at high Froude numbers. Trimarans share most of the characteristics of catamarans, but in few aspects, trimarans are more efficient than catamarans. Lyakhovitsky compared a trimaran with a mono-hull and a catamaran of same characteristics and showed that the trimaran is better in hydrodynamic performances compared to other alternatives [1]. In addition trimarans have some other privileges such as: extended deck, lower draft and better transverse stability compared with single body vessels [2]. In order to study the effect of outriggers position on trimaran resistance, some experimental tests are done and results show that the outriggers location has considerable effect in hydrodynamic performance of the vessel [3], but in vessel design, some cases such as maneuverability must be consider.

Optimization procedures demand the performance of a ship to be assessed in its early design stage. This leads to a prediction tool independent of experimental results, although model tests will still be indispensable. CFD modeling based on numerical solution of the governing equations is a good choice. It must be remembered that, such a problem combines the complexity of free surface flow with rigid body motions. NUMEL ${ }^{1}$ code [4], [15] which is used for present study provides an effective numerical tool for hydrodynamic simulation. Trimaran maneuvering simulation is a complex hydrodynamic problem that should be divided into minor sub-problems.

The motion of a floating body is a direct consequence of the flow-induced forces acting on it while at the same time these forces are functions of the body movement itself. Therefore, the prediction of flow-induced body motion in viscous fluid is a challenging task and requires coupled solution of fluid flow and body motions. In recent two decades, with the changes in computer hardware, ship motion simulation is the subject of many numerical hydrodynamic researches. These researches were started from the restricted motions such as trim or sinkage by Miyata [5], Hochbaum [6] Alessandrini [7] and Kinoshita [8] and continued to the evaluation of 6-DoF motions by Miyake [9], Azcueta [10], Vogt [12], Xing [12] and Jahanbakhsh et. al [13]. In this paper fully nonlinear motion of Trimaran is simulated based on 6-DOF motions and hydrodynamic interaction.

\footnotetext{
${ }^{1}$ Numerical Marine Engineering Laboratory
} 


\section{GOVERNING EQUATIONS}

There is an approach in simulation of two-phase flow where different fluids are modeled as a single fluid obeying the same set of governing equations, with the different local identified volume fraction values $\alpha$. Incompressible NavierStokes and continuity equations are well-known and given by the equations:

$$
\begin{gathered}
\frac{\partial u_{i}}{\partial t}+u_{j} \frac{\partial u_{i}}{\partial x_{j}}=-\frac{1}{\rho} \frac{\partial P}{\partial x_{i}}+\frac{\partial^{2} u_{i}}{\partial x_{j} \partial x_{j}}+g_{i} \\
\frac{\partial u_{i}}{\partial x_{i}}=0
\end{gathered}
$$

where:

$\mathrm{u}_{\mathrm{i}}-$ velocity

$\mathrm{P}^{1}-$ pressure

$v-$ kinematic viscosity.

Local density $\rho$ and viscosity $v$ of the single fluid are defined as:

$$
\begin{aligned}
\rho_{\text {cell }} & =\alpha \rho_{1}+(1-\alpha) \rho_{2} \\
\text { cell } & =\alpha{ }_{1}+(1-\alpha){ }_{2}
\end{aligned}
$$

Subscripts 1 and 2 indicate two fluids (e.g. water and air), where $\alpha$ (volume fraction) is the percentage of fluid 1 (e.g. water) available in cell and defined as follow:

$$
\alpha=\left\{\begin{array}{cc}
1 & \text { for cells inside fluid } 1 \\
0 & \text { for cells inside fluid } 2 \\
0<\alpha_{0}<1 & \text { for transitional area }
\end{array}\right.
$$

Reformulating the continuity equation (Eq.2) and using the definition of the single fluid density, results in extracting a scalar transport equation for volume fraction $\alpha$ (Spalding, 1974):

$$
\frac{\partial \alpha}{\partial \mathrm{t}}+\vec{\nabla} \cdot(\alpha \overrightarrow{\mathrm{u}})=0
$$

Discretisation of the governing equations is considered by integration of the momentum equation over a control volume it becomes as below:

$$
\begin{gathered}
\frac{\mathrm{d}}{\mathrm{dt}} \int_{\mathrm{V}} \overrightarrow{\mathrm{u}} \mathrm{dV}+\int_{\mathrm{A}} \overrightarrow{\mathrm{u}}(\overrightarrow{\mathrm{u}} \cdot \overrightarrow{\mathrm{n}}) \mathrm{dA}= \\
=\int_{\mathrm{A}} v \vec{\nabla} \overrightarrow{\mathrm{u}} \cdot \overrightarrow{\mathrm{n}} \mathrm{dA}-\frac{1}{\rho} \int_{\mathrm{A}} \mathrm{P} \overrightarrow{\mathrm{n}} \mathrm{dA}+\int_{\mathrm{V}} \overrightarrow{\mathrm{g}} \mathrm{dV}
\end{gathered}
$$

Where: $\overrightarrow{\mathrm{u}}$ is the velocity vector, $v$ is the cell volume and A is the area around it.

The diffusion term (the first term in r.h.s. of Eq.6) is discretised using the over-relaxed interpolation for velocity component $u_{i}$ (Jasak, 1996):

$$
\int_{\mathrm{A}} v \vec{\nabla} \overrightarrow{\mathrm{u}}_{\mathrm{i}} \cdot \overrightarrow{\mathrm{n}} \mathrm{dA}=\sum_{\mathrm{f}=1}^{\mathrm{n}} \mathrm{v}_{\mathrm{f}} \overrightarrow{\mathrm{A}}_{\mathrm{f}} \cdot\left(\vec{\nabla} \overrightarrow{\mathrm{u}}_{\mathrm{i}}\right)_{\mathrm{f}}
$$

Where: $\overrightarrow{\mathrm{A}}_{\mathrm{f}}$ is the $\mathrm{CV}$ face area vector.

Discretisation of the convection term (the second term in 1.h.s. of Eq.6) needs to the fluid velocity component on $\mathrm{CV}$ face $u_{i-f}$ as shown in Eq.8:

$$
\int_{A} u_{i}(\vec{u} \cdot \vec{n}) d A=\sum_{f=1}^{n} u_{i-f} F_{f}
$$

Where: $F_{f}=\vec{A}_{f} \cdot \vec{U}_{f}$ is the volumetric flux. The fluid velocity on $\mathrm{CV}$ face $\overrightarrow{\mathrm{U}}_{\mathrm{f}}$ must be calculated separately in the co-located arrangement to avoid checkerboard pressure and will be discussed later in the solution algorithm. Here, $\mathrm{u}_{\mathrm{i}-\mathrm{f}}$ is approximated using Gamma interpolation scheme (Jasak, 1996) based on NVD (Normalized Variable Diagram) (Leonard, 1991) concept:

$$
\mathrm{u}_{\mathrm{i}-\mathrm{f}}=\left\{\begin{array}{cl}
\mathrm{u}_{\mathrm{i}-\mathrm{D}} & \text { for } \tilde{\mathrm{u}}_{\mathrm{i}-\mathrm{D}} \leq 0 \text { or } \tilde{\mathrm{u}}_{\mathrm{i}-\mathrm{D}} \geq 1 \\
\frac{1}{2}\left(\mathrm{u}_{\mathrm{i}-\mathrm{D}}+\mathrm{u}_{\mathrm{i}-\mathrm{A}}\right) & \text { for } \mathrm{k} \leq \tilde{\mathrm{u}}_{\mathrm{i}-\mathrm{D}}<1 \\
\left(1-\frac{\mathrm{u}_{\mathrm{i}-\mathrm{D}}}{2 \mathrm{k}}\right) \mathrm{u}_{\mathrm{D}}+\frac{\mathrm{u}_{\mathrm{i}-\mathrm{D}}}{2 \mathrm{k}} \mathrm{u}_{\mathrm{i}-\mathrm{A}} & \text { for } 0 \leq \tilde{\mathrm{u}}_{\mathrm{i}-\mathrm{D}}<\mathrm{k}
\end{array}\right.
$$

Subscripts D and A stand for donor and acceptor cells determined for each CV's face according to the direction of flow as shown in Fig. 1. In addition, $\phi_{\mathrm{D}}$ and $\phi_{\mathrm{f}}$ are defined based on NVD as Eqs.10 and 11.

$$
\begin{gathered}
\phi_{\mathrm{D}}=\frac{\phi_{\mathrm{D}}-\phi_{\mathrm{U}}}{\phi_{\mathrm{A}}-\phi_{\mathrm{U}}} \\
\phi_{\mathrm{f}}=\frac{\phi_{\mathrm{f}}-\phi_{\mathrm{U}}}{\phi_{\mathrm{A}}-\phi_{\mathrm{U}}}
\end{gathered}
$$

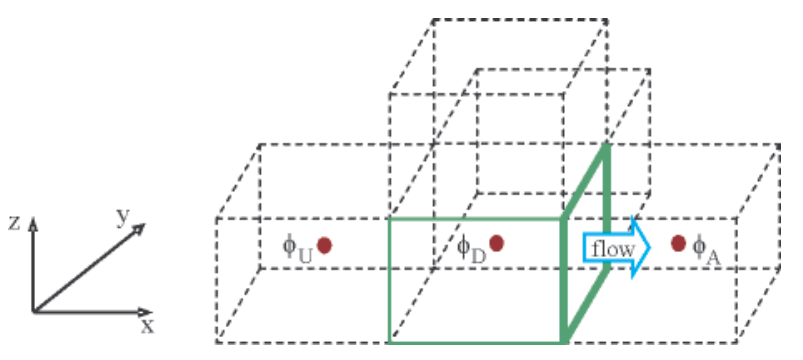

Fig. 1. Flow direction (arrow) determines doner, acceptor and upwind cells for each CV's face

It must be mentioned that the Crank-Nicholson scheme is used for time discretisation of diffusion and convection terms in momentum equation (Eq.6). The pressure term (second term in r.h.s. of Eq.6) is discretised as Eq.12:

$$
\int_{\mathrm{A}} \mathrm{P} \overrightarrow{\mathrm{n}} \mathrm{dA}=\sum_{\mathrm{f}=1}^{\mathrm{n}} \mathrm{P}_{\mathrm{f}} \overrightarrow{\mathrm{A}}_{\mathrm{f}}
$$

Using the common Linear Interpolations (LI) for calculation of face pressure $P_{f}$, results in severe oscillations in velocity field. This is of great importance, especially when there are two fluids with high density ratio e.g. water and air. Here a Piecewise Linear Interpolation (PLI) shown in Fig. 2 is introduced and used for $\mathrm{P}_{\mathrm{f}}$ estimation. It is based on a constraint for lines $\mathrm{L}_{\mathrm{Af}}$ and $\mathrm{L}_{\mathrm{Bf}}$ which connect pressure values at CVs' center $\mathrm{P}_{\mathrm{A}}$ and $\mathrm{P}_{\mathrm{B}}$ to $\mathrm{P}_{\mathrm{f}}$ as Eq.13:

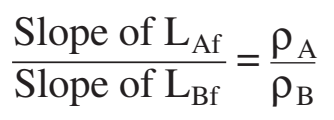

Where $\rho_{A}$ and $\rho_{B}$ are the densities of CVs A and B, respectively. Therefore $P_{f}$ can be estimated by using the pressure value at CVs' center $\mathrm{P}_{\mathrm{A}}$ and $\mathrm{P}_{\mathrm{B}}$ as well as Eq.14:

$$
\mathrm{P}_{\mathrm{f}}=\mathrm{P}_{\mathrm{A}} \kappa+\mathrm{P}_{\mathrm{B}}(1-\kappa)
$$

$\kappa$ is the weighting factor and can be calculated as Eq.15:

$$
\kappa=\frac{\rho_{B} \delta_{B}}{\rho_{A} \delta_{A}+\rho_{B} \delta_{B}}
$$


Where $\delta_{A}$ and $\delta_{B}$ are distance from face center $f$ to CVs' center A and B, respectively (Fig. 2). Finite volume discretisation of volume fraction transport equation (Eq.5) is based on the integration over $\mathrm{CV}$ and time step:

$$
\int_{\mathrm{t}}^{\mathrm{t}+\delta \mathrm{t}}\left(\int_{\mathrm{V}} \frac{\partial \alpha}{\partial \mathrm{t}} \mathrm{dV}\right) \mathrm{dt}+\int_{\mathrm{t}}^{\mathrm{t}+\delta \mathrm{t}}\left(\int_{\mathrm{V}} \vec{\nabla} \cdot(\alpha \overrightarrow{\mathrm{u}}) \mathrm{dV}\right) \mathrm{dt}=0
$$

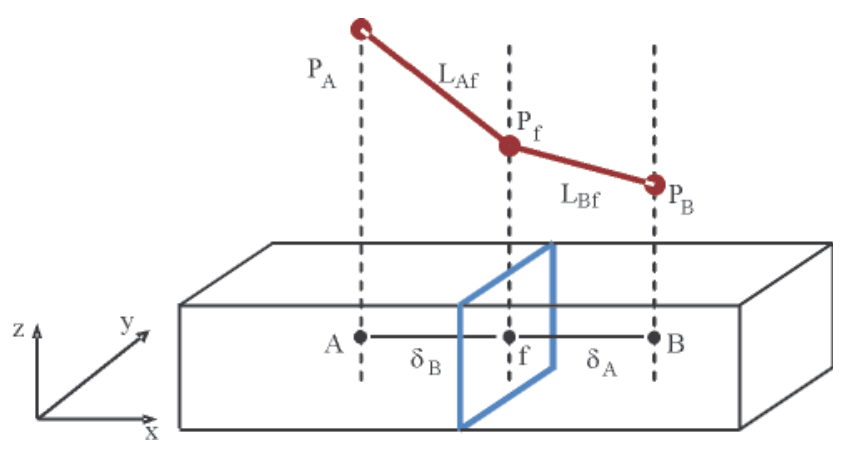

Fig. 2. PLI for CV's face pressure calculation

The first term in Eq.16 is a common integral form and applying the Gauss theorem on the second term results in:

$\left(\alpha_{\mathrm{P}}^{\mathrm{t}+\delta \mathrm{t}}-\alpha_{\mathrm{P}}^{\mathrm{t}}\right) \frac{\mathrm{V}}{\delta \mathrm{t}}+\frac{1}{2}\left(\sum_{\mathrm{f}=1}^{\mathrm{n}} \alpha_{\mathrm{f}}^{\mathrm{t}+\delta \mathrm{t}} \mathrm{F}_{\mathrm{f}}^{\mathrm{t}+\delta \mathrm{t}}+\sum_{\mathrm{f}=1}^{\mathrm{n}} \alpha_{\mathrm{f}}^{\mathrm{t}} \mathrm{F}_{\mathrm{f}}^{\mathrm{t}}\right)=0$

The time integral of the second term is discretised using Crank-Nicholson scheme. Assuming a linear and small variation of $F_{f}$ in small time step, results in using the most recent value of it. Taking this into account, and rearranging of Eq. 17 yield to:

$$
\alpha_{P}^{t+\delta t} \frac{V}{\delta t}+\sum_{f=1}^{n} \frac{1}{2} \alpha_{f}^{t+\delta t} F_{f}=S_{\alpha_{P}}
$$

Where the source term is:

$$
S_{\alpha_{P}}=\alpha_{P}^{t} \frac{V}{\delta t}-\sum_{f=1}^{n} \frac{1}{2} \alpha_{f}^{t} F_{f}
$$

One can see the face values $\alpha_{f}$ which must be approximated using an interpolation. As aforementioned, simple interpolations leads to non-physical or too diffusive volume fraction values. This leads to use a high order composite one. Most of composite methods, typically switch between two high and low order interpolations to use their advantages. Here, the main distinctions are how and when they switch between these schemes according to flow information.

CICSAM uses CBC (Convection Boundedness Criteria) (Gaskell and Lau, 1988) and UQ (ULTIMATE-QUICKEST) (Leonard, 1991) by introducing a weighting factor $\gamma_{\mathrm{f}}$ (Eq.20) which takes into account the slope of the free surface relative to the direction of motion. $\mathrm{CBC}$ is the most compressive scheme that stipulates robust local bounds on $\tilde{\alpha}_{f}$ nevertheless does not actually preserve the shape of interface. Here UQ uses for its ability to better preserving of interface shape. Based on NVD, normal face value is obtained as follows:

$$
\tilde{\alpha}_{f}=\gamma_{f} \tilde{\alpha}_{f_{C B C}}+\left(1-\gamma_{f}\right) \tilde{\alpha}_{f_{U Q}}
$$

Using the definition of Eq.11 in Eq.20, results in estimation of $\alpha_{\mathrm{f}}$, shown in Eq.18. This value contains all the information regarding to the fluid distribution in the donor, acceptor and upwind cells as well as the interface orientation relative to flow direction. To avoid non-physical $\alpha$ in highly skewed meshes, a correction step is added to volume fraction calculation procedure and used in the developed software which can be found in Ubbink and Issa (1999) by details. Fig. 3 shows the solution algorithm in the developed numerical tool.

\section{NUMERICAL RESULTS}

\section{Trimaran Resistance}

Although many simulations have been done to investigate the accuracy of this software and all of the results were in good agreement with experimental data $[4,15]$, but still in present study, the accuracy of the code is validated by simulating a trimaran vessel and comparing the numerical result with experimental result.

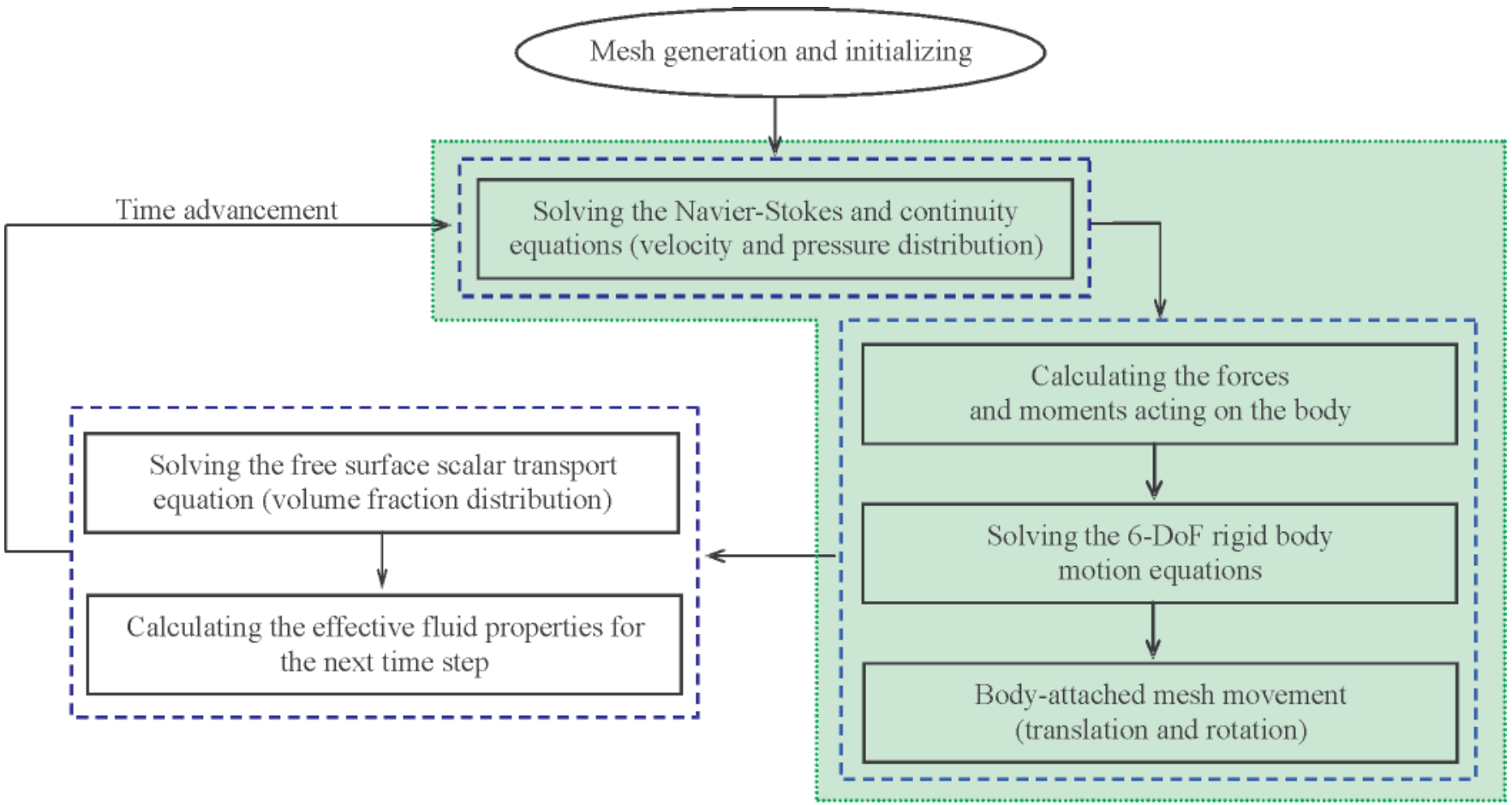

Fig. 3. Solution algorithm in the developed numerical tool 
The trimaran with wigley form has been chosen; the main geometric characteristics of trimaran are given in Table 1. These characteristics are similar to that used in the experimental tests at Naples and Trieste Universities [14]. The computational domain dimensions are $28 \times 14 \times 3$ meters $(7$ meters in front of vessel, 13.3 in behind of the vessel, 1 meter upside the water line and 2 meters under the keel line). In this software, the hexagonal meshes are used. For resistance simulation, since trimaran is an axis-symmetric body, because of symmetric shape of the hull, only half of the domain is used to reduce calculation as shown in Fig. 4. The numbers of cells in this domain are about 120000. This vessel has been simulated for speed range of $4 \sim 6 \mathrm{~m} / \mathrm{s}$. Since longitudinal location (the direction of motion) has more influence on the resistance than transverse location, the effect of four longitudinal and two transverse locations of outriggers have been studied as shown in Table 2. The parameters are defined in Tables 1 and 2, and illustrated in Fig. 5. The trimaran configuration is defined by the ratios $d / \mathrm{L}_{M}$ and $\mathrm{s} / \mathrm{L}_{\mathrm{M}}$, where $\mathrm{d}$ is the longitudinal distance between the bows of the main hull and the outriggers, and $\mathrm{s}$ is the transverse distance between the centerline of outriggers. In Table 2 and Fig. 5, the under notes $\mathrm{M}$ and $\mathrm{O}$ are pointed to main hull and outriggers respectively. In order to validate simulation results, initially a simulation similar to the one studied in reference [16] is performed and then the results are compared, Fig. 6.

Table 1. Trimaran particulars

\begin{tabular}{|c|c|c|}
\hline & Main Hull & Side Hull \\
\hline Length waterline $(\mathrm{m})$ & 4.694 & 2.347 \\
\hline Draught $(\mathrm{m})$ & 0.166 & 0.0463 \\
\hline Wetted surface $\left(\mathrm{m}^{2}\right)$ & 1.948 & 0.252 \\
\hline Displacement $(\mathrm{kg})$ & 120.489 & 4.259 \\
\hline Beam waterline $(\mathrm{m})$ & 0.332 & 0.109 \\
\hline
\end{tabular}

Table 2. Trimaran configurations

\begin{tabular}{|c|c|c|c|c|}
\hline $\mathbf{d} / \mathbf{L m}$ & $\mathbf{- 0 . 2 5}$ & $\mathbf{0 . 0}$ & $\mathbf{+ 0 . 5}$ & $+\mathbf{0 . 7 5}$ \\
\hline 0.2 & $\mathrm{~A}$ & $\mathrm{~B}$ & $\mathrm{C}$ & $\mathrm{D}$ \\
\hline & - & $\mathrm{E}$ & $\mathrm{F}$ & - \\
\hline
\end{tabular}

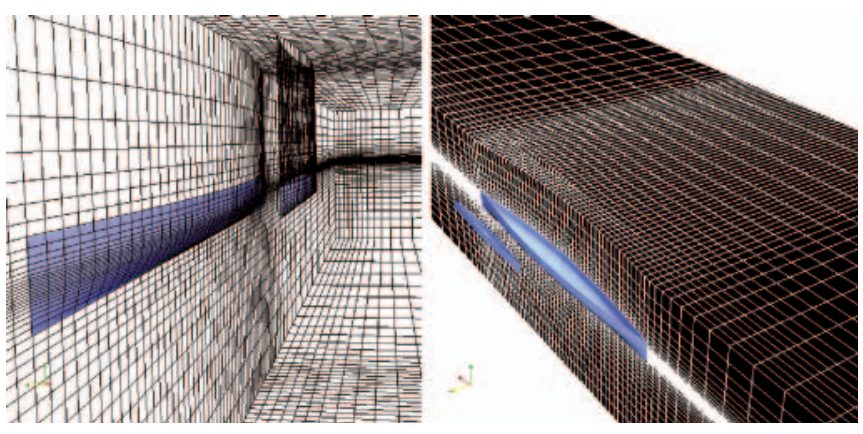

Fig. 4. Computational domain

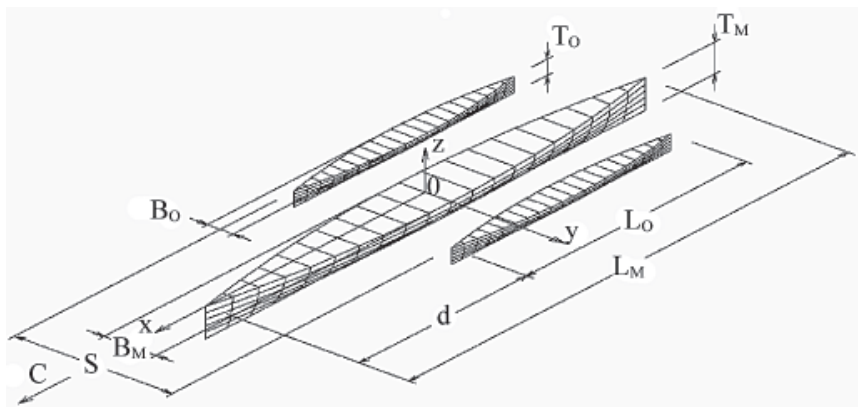

Fig. 5. trimaran configuration with main particulars and relative position of outriggers

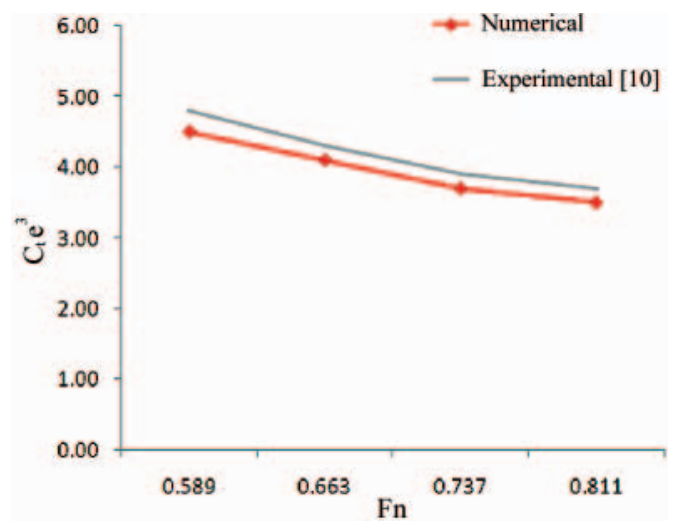

Fig. 6. Comparison between numerical results and experimental data for case $C$

Fig. 7 represents pressure drag relative to vessel speed for four cases with same clearance. For speed less than about 4.3 $\mathrm{m} / \mathrm{s}$, case A has the least pressure drag and for speed more than approximately 4.3 , case D has the least pressure drag. The case $\mathrm{B}$ has the largest pressure drag for speed less than $5 \mathrm{~m} / \mathrm{s}$ and at speeds more than $5 \mathrm{~m} / \mathrm{s}$, the case $\mathrm{C}$ has the largest pressure drag. Viscous drag versus speed is shown in Fig. 8 and also shows that case $\mathrm{A}$ has the least viscous drag. The case $\mathrm{B}$ has highest viscous drag for speed less than $5.1 \mathrm{~m} / \mathrm{s}$ and for speed more than $5.5 \mathrm{~m} / \mathrm{s}$ it is close to configuration A, but as the speed increases, the case $\mathrm{C}$ has the most viscous drag. Generally, the optimal viscous and pressure drag depend on vessel speed. In other words, the vessel speed must be considered in order to obtain the appropriate trimaran configuration which has the least pressure or viscous drag. It can be seen from Fig. 9 that trimaran with configuration A has the least of total drag (Sum of viscous and pressure drag). In this configuration, the waves created by main body do not interact with side bodies. This phenomenon can be accounted as a reason drag reduction. Fig. 10 represents trimaran with $\mathrm{A}$ configuration at speed $4 \mathrm{~m} / \mathrm{s}$. So it is seen that at higher speeds, because of decreasing of wave propagation angle, the wave created by main body do not interact with side bodies.

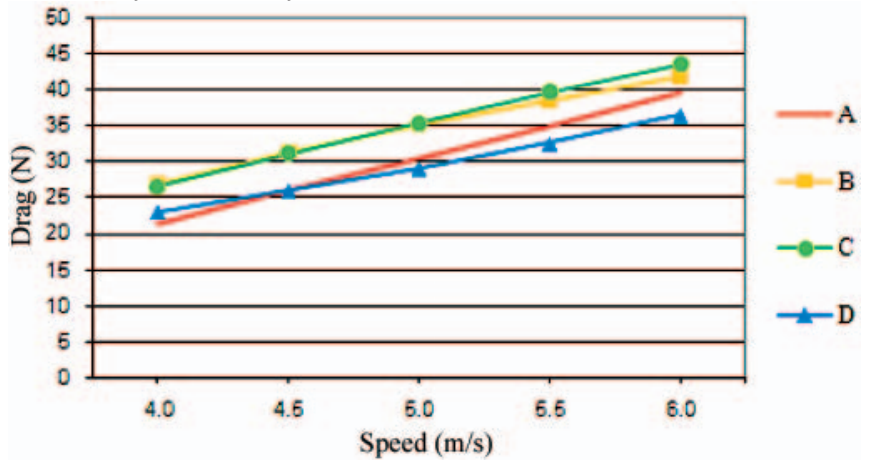

Fig. 7. Pressure drag for various configurations

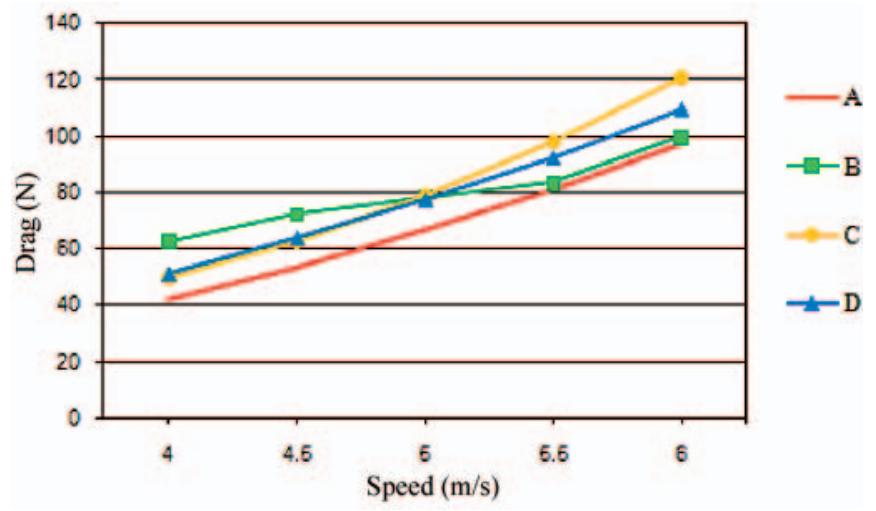

Fig. 8. Viscous drag for various configurations 


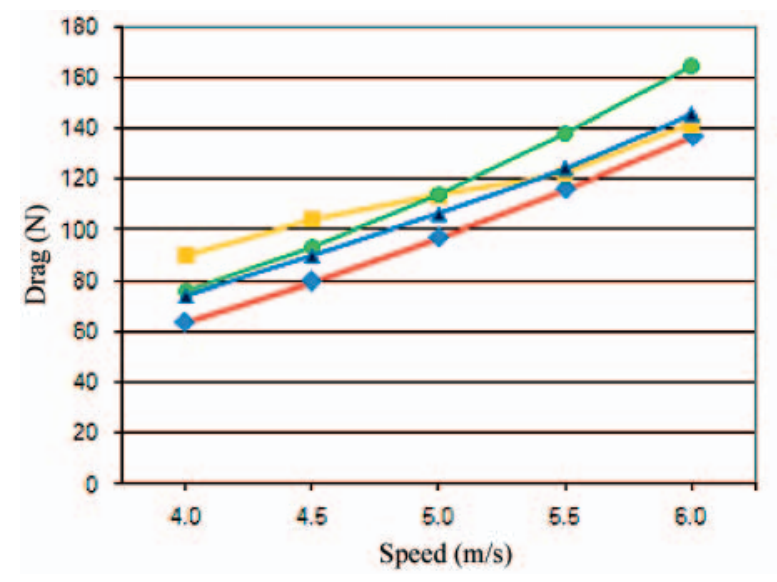

Fig. 9. Total drag for various configurations

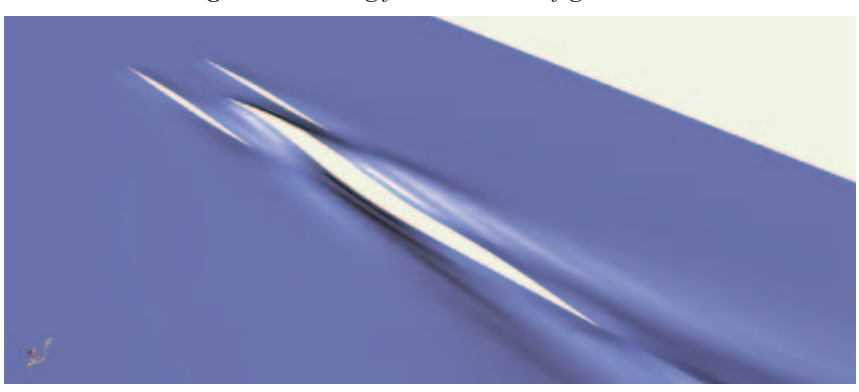

Fig. 10. Free surface for case A at speed $4 \mathrm{~m} / \mathrm{s}$

At higher speeds, the rate of increasing total drag in configuration B decreases. As the speed increases, the wave propagation angle decreases, so the interaction length between waves created by main body with the side bodies decrease. It is seen from Fig 11-A and 11-B, that at speed of $4 \mathrm{~m} / \mathrm{s}$, waves created by main body interacts with about $3 / 4$ length of side bodies, whereas at speed $5.5 \mathrm{~m} / \mathrm{s}$, they affected only the transom of side bodies. In order to validate the above statement, trimaran with configurations $\mathrm{E}$ and $\mathrm{F}$ are simulated. Fig. 12 shows pressure drag. It is observed that with increasing transverse distance, pressure drag decreases. Comparison of configuration $\mathrm{B}$ and $\mathrm{E}$, showed that viscous drag in configuration $\mathrm{E}$ is less than configuration $\mathrm{B}$, but increase in transverse distance has inverse effect when stern of bodies are aligned (compare configurations $\mathrm{C}$ and $\mathrm{F}$ ). Fig. 13 shows viscous drag. In Fig. 14 it is observed that for configuration E, even at speed of
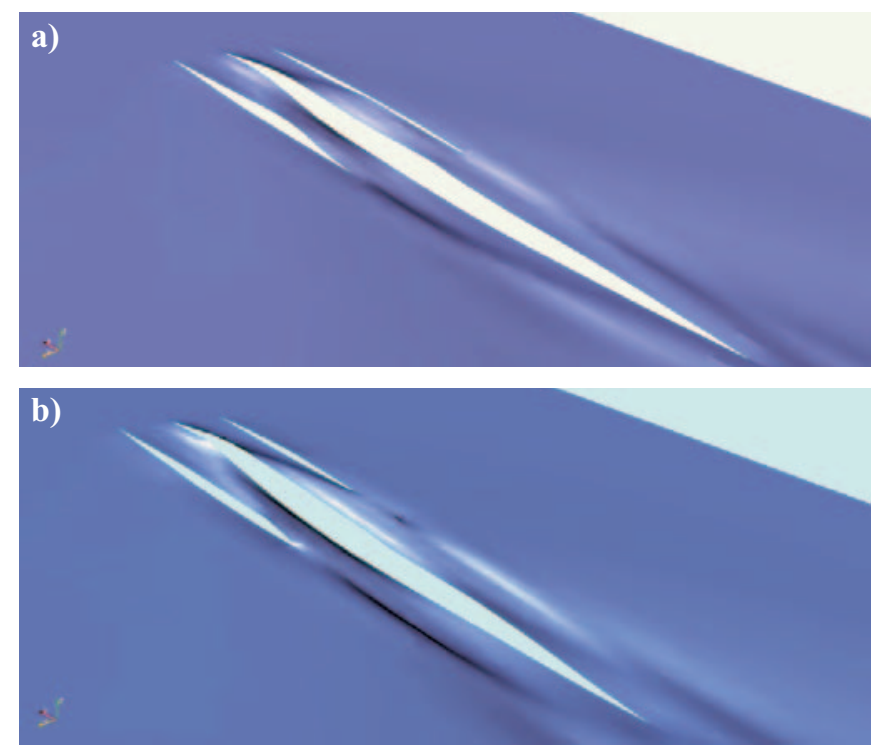

Fig. 11. Free surface for configuration $B$. a) at speed $4 \mathrm{~m} / \mathrm{s}$, b) at speed $5.5 \mathrm{~m} / \mathrm{s}$
$4 \mathrm{~m} / \mathrm{s}$ main hull waves do not interact with outriggers but with increasing separation when stern of bodies are aligned, more waves pass through the tunnel between bodies, as a result the wave interaction increases, and consequently, as a result of increase in wet surface, viscous drag increases in configuration $\mathrm{F}$ with respect to configuration $\mathrm{C}$. Finally, increase in transverse distance causes drag reduction when three hulls bow are aligned, but does not affect when three hulls stern are aligned (see Fig. 15).

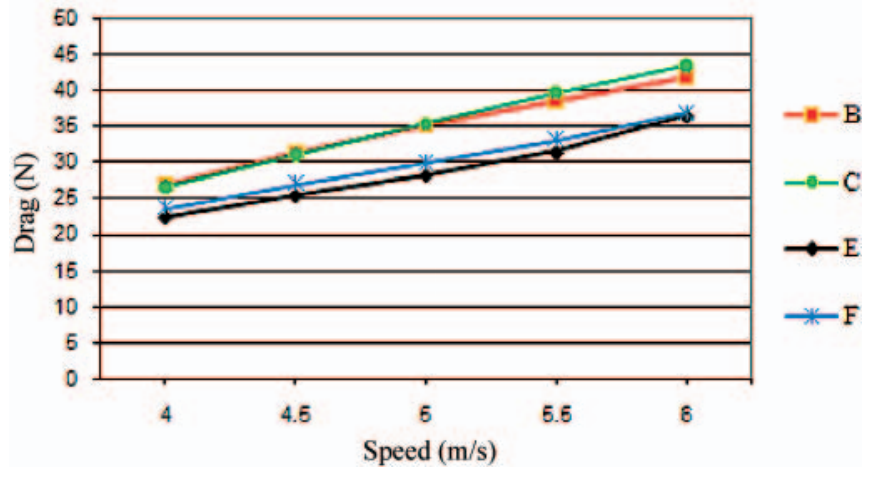

Fig. 12. Pressure drag for various configurations

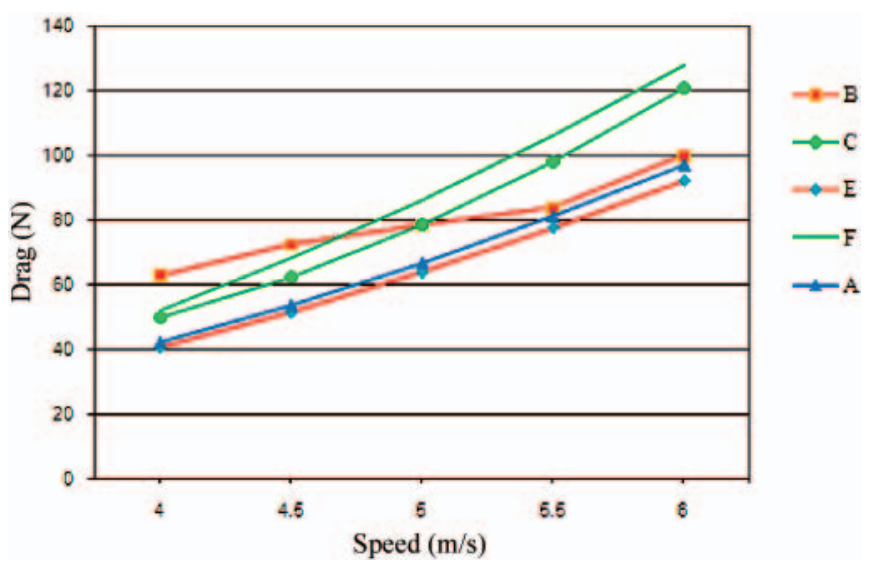

Fig. 13. Viscous drag for various configurations

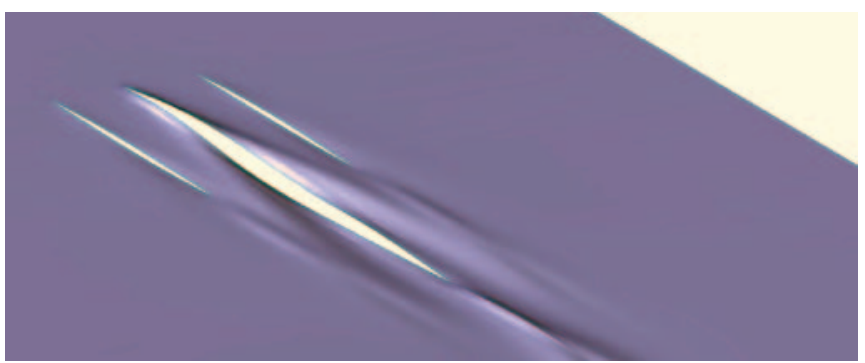

Fig. 14. Free surface for configuration $E$ at speed $4 \mathrm{~m} / \mathrm{s}$

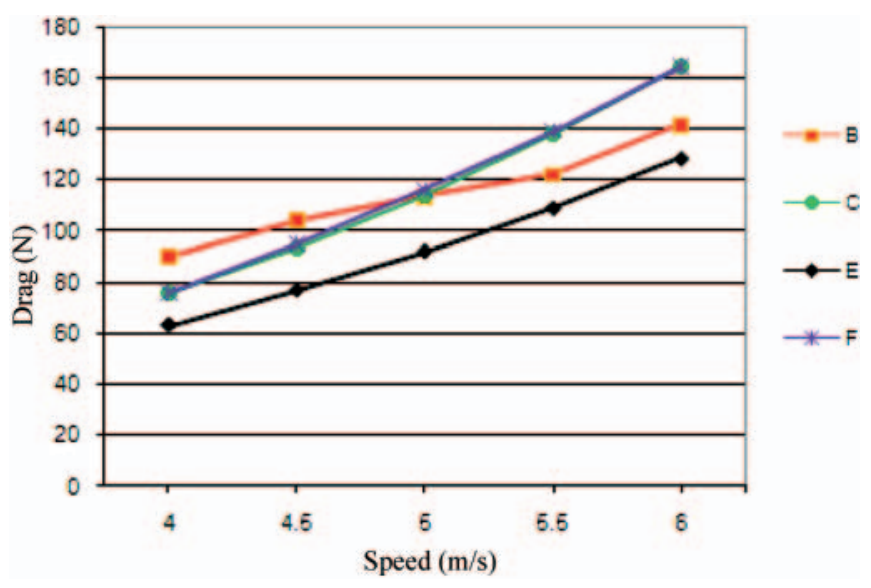

Fig. 15. Total drag for various configurations 


\section{Trimaran Maneuvering}

For study of outriggers position on trimaran maneuvering, the effects of three longitudinal positions of outriggers are investigated. Since motions in maneuvering are not symmetric, it is not possible to use half domain for simulating, so in maneuvering simulation used wigley trimaran that is smaller than that used for resistance study to reduce the calculation. Table 3 and Table 4 present the characteristics and configurations of this trimaran. Applying forces and moments of maneuvering is performed by rotation of thrusters. So there is no rudder here and whole of propulsion system assumed to be rotated. Angle of rotation which applied on the trimaran's propulsion systems is 30 degrees. It should be noted that propulsion system assemble on the main hull and turning starts just after 10 seconds from the beginning of simulation. This permits the ship to reach a nearly steady forward motion due to thrusters' force. At first, various trimaran configurations are simulated at $4 \mathrm{~m} / \mathrm{s}$ speed and required force to reach this speed is calculated which is shown in Table 5. It is clear that for each configuration the thruster force is equal to its corresponding total resistance forces.

Table 3. Trimaran characteristics

\begin{tabular}{|c|c|c|}
\hline & Main Hull & Side Hull \\
\hline Length L(m) & 2.4 & 1.2 \\
\hline Breadth B(m) & 0.24 & 0.12 \\
\hline Draft $\mathrm{T}(\mathrm{m})$ & 0.15 & 0.075 \\
\hline Displacement $(\mathrm{kg})$ & 40.0 & 5.0 \\
\hline Wetted surface $\left(\mathrm{m}^{2}\right)$ & 0.88 & 0.44 \\
\hline
\end{tabular}

Table 4. Trimaran configurations

\begin{tabular}{|c|c|c|c|}
\hline $\mathrm{d} / \mathrm{Lm}$ & 0 & 0.25 & 0.5 \\
\hline 0.2 & $\mathrm{~A}$ & $\mathrm{~B}$ & $\mathrm{C}$ \\
\hline
\end{tabular}

Table 5. Total drag at $4 \mathrm{~m} / \mathrm{s}$ speed for different configuration

\begin{tabular}{|l|c|c|c|}
\hline Configuration & A & B & C \\
\hline Total drag $(\mathrm{N})$ & 43.92 & 55.00 & 48.24 \\
\hline
\end{tabular}

Fig. 16 shows the time history of ship speed for different configurations. It can be seen that for A configuration decreasing speed at turning is more than other configurations. Path of ship's center of gravity is shown in Fig. 17. In the turning circle, the diameter of rotation circle for A configuration is most magnitude and for $\mathrm{B}$ and $\mathrm{C}$ configuration is close together. In Fig. 18 trim angle of the vessel are shown. It is obvious that when the side bodies stem are aligned with main hull stem, the vessel trim is more than $\mathrm{B}$ and $\mathrm{C}$ configuration and when the three bodies stern of vessel are aligned (C configuration), trimaran has least trim angle. Therefore when the outriggers are in front of vessel (A configuration), it leads to a large trim angle which causes some section of side hulls come out of water and therefore stability decreases. In Fig. 19, heel angle of various trimaran configurations is plotted. It can be seen that A configuration has least heel angle and its magnitude is near 1.5 degree, but oscillation magnitude is more than other configurations. Drift angle of three different configurations is also plotted in Fig. 20. It is clear that trimaran with A configuration has not stable drift angle. Fig. 21 shows Time history of yaw speed. It can be seen that A configuration has lowest yaw speed. Least yaw speed and unstable drift angle for A configuration can be reason of largest diameter of turning circle relate to other configurations. Figs 22 24 includes few snapshots of trimaran and free surface around it during turning maneuver. The unsymmetrical waves generated during turning can be seen in this figure.

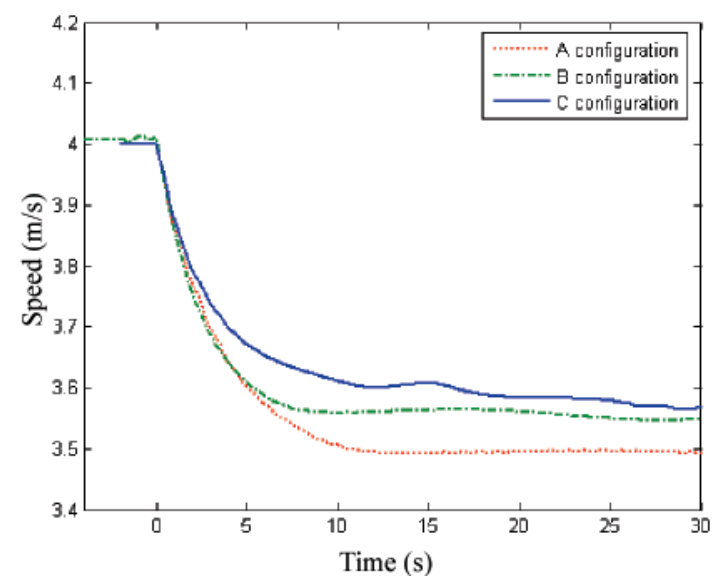

Fig. 16. Speed time history for different configurations

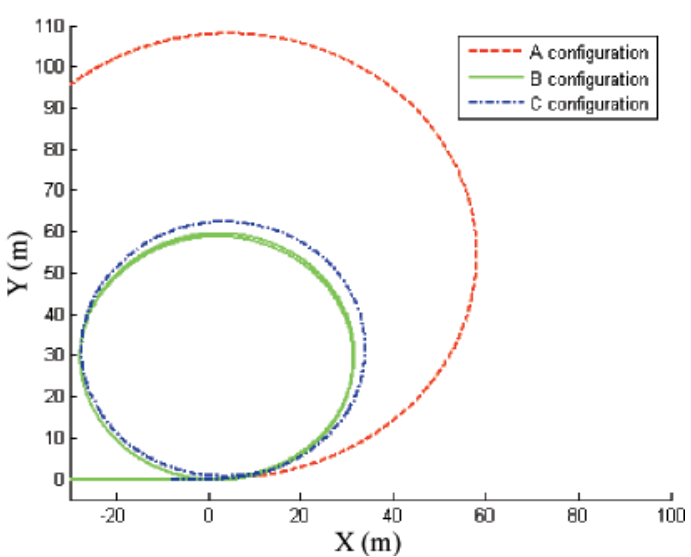

Fig. 17. Ship mass center path

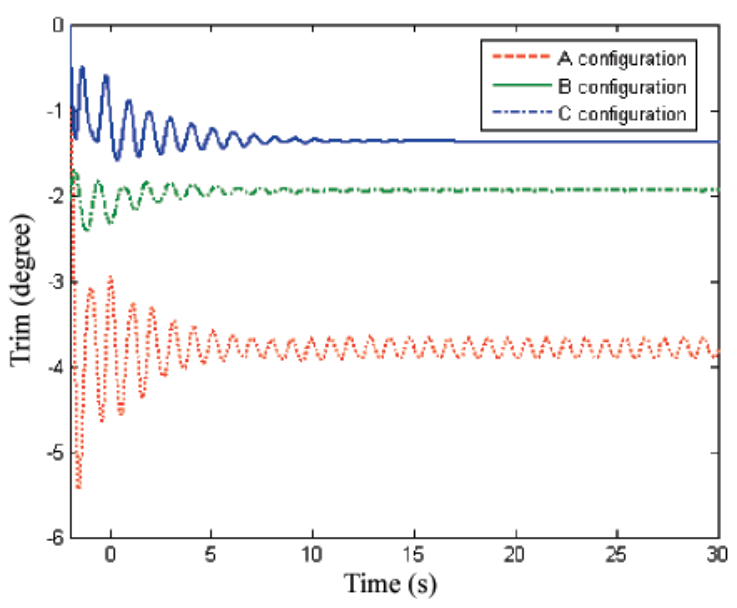

Fig. 18. Trim angle time history for different configuration

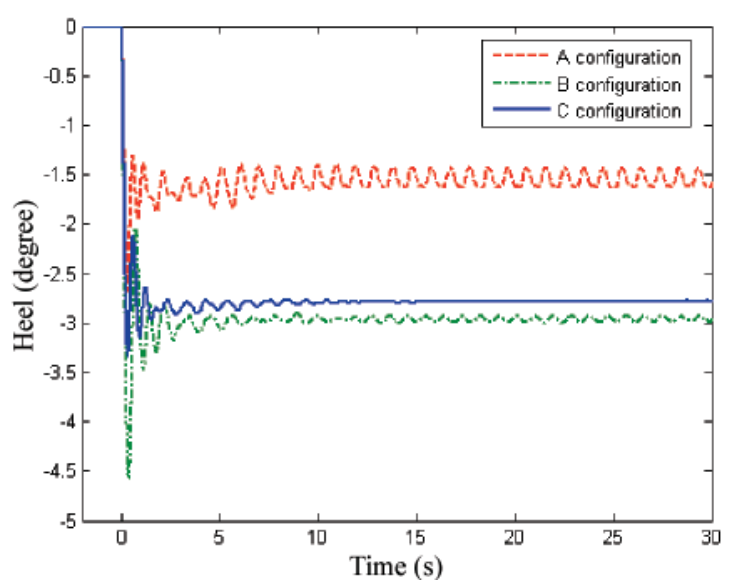

Fig. 19. Heel angle time history for different configurations 


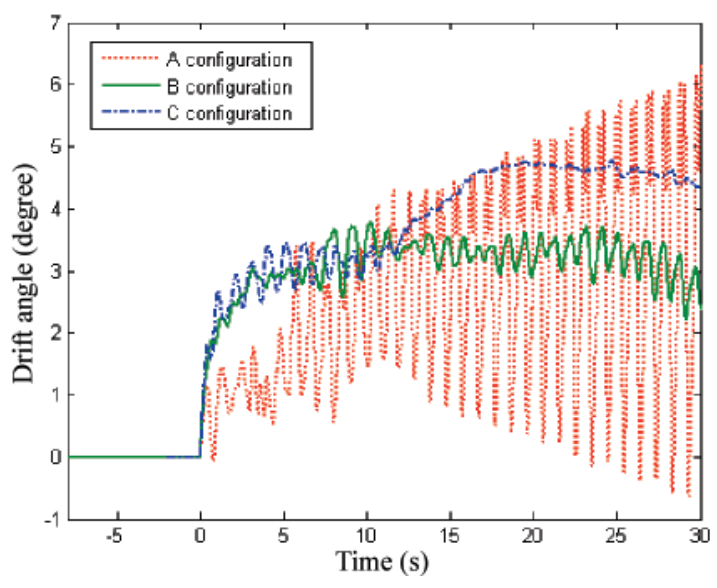

Fig. 20. Drift angle time history for different configuration

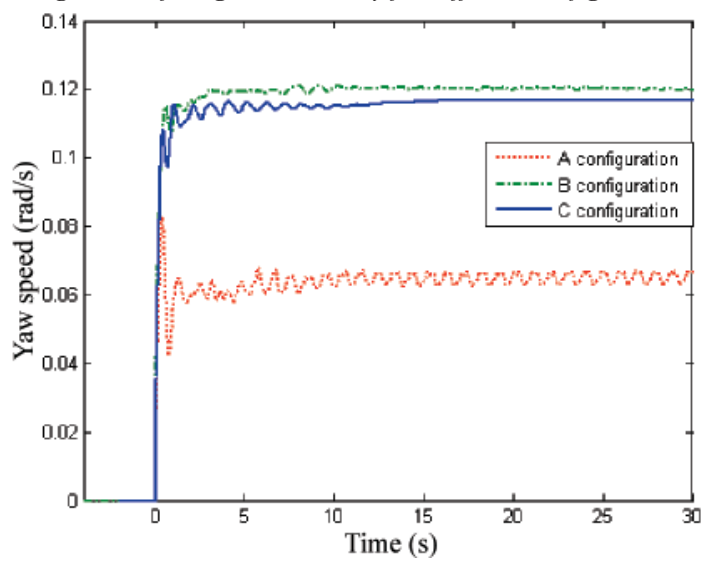

Fig. 21. Yaw speed time history for different configuration

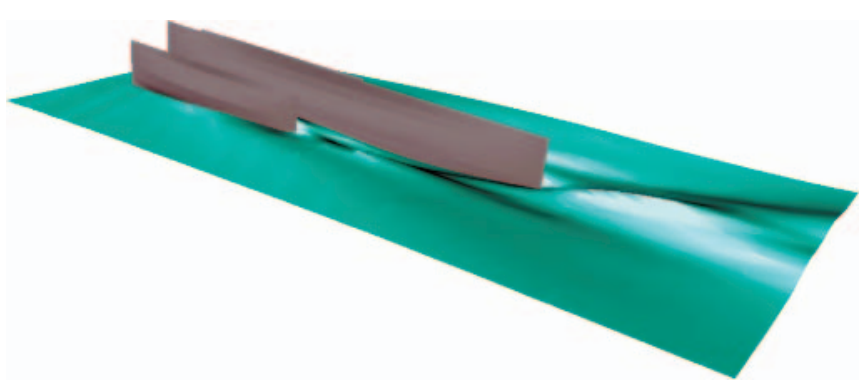

Fig. 22. Free surface for A configuration

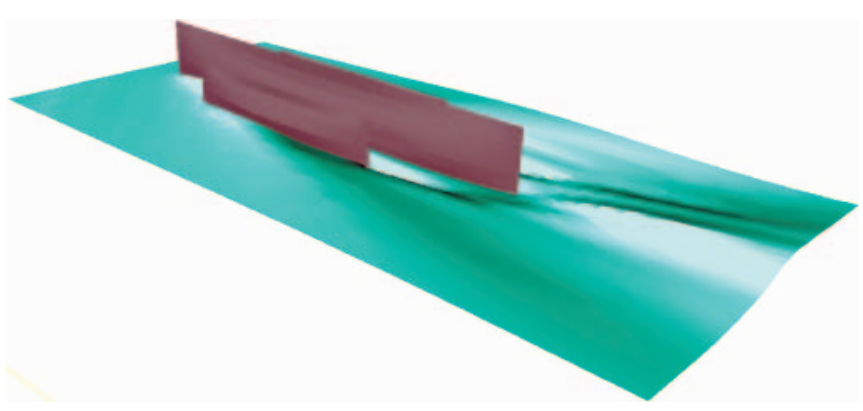

Fig. 23. Free surface for B configuration

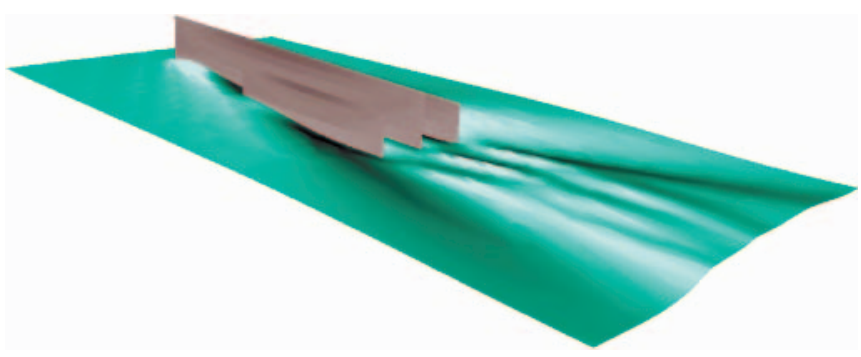

Fig. 24. Free surface for C configuration

\section{CONCLUSION}

In this paper, the effects of outriggers location on trimaran hydrodynamic resistance and maneuverability are studied. Vessel body form is considered as standard wigley series and it is analyzed by numerical simulation scheme. In order to generalize the results, simulations for wide speed range are necessary, but based on the simulations performed the following conclusions can be drawn.

For trimaran configuration when the three bow hulls are aligned, as the speed increases, the rate of total drag growth will decrease, because the length of interaction between the waves created by the main hull with outriggers decreases. Resistance decreases with increasing transverse distance where three hulls bow are aligned, but increasing transverse distance does not affect where three bodies stern are aligned.

O Finally, it should be taken into account that hydrodynamic resistance is an important factor for trimaran design, but factors such as maneuverability and seakeeping are also considerable. Maneuvering of a trimaran vessel has been also investigated in present paper taking into account 6-DoF rigid body motion. Numerical results show that outriggers position has great effect on trimaran maneuverability. Based on these results, it can be seen that when the bow of three bodies are aligned, the maneuvering quality is not good, because in this case, vessel trim causes outriggers to come out from water. Therefore motion stability decreases. Other configurations have almost the same turning circles diameter. But from resistance point of view, when the stern of three hulls are aligned, less thrust force is necessary, so it is more effective than other configurations.

\section{BIBLIOGRAPHY}

1. V. Dubrovsky \& A. Lyakhovitsky, Mullty Hull Ships, Backbone Publishing,USA, (2001)

2. N. Degiuli, A. Werner, I. Zotti, an experimental investigation into the resistance components of Trimaran configurations, FAST (2005)

3. K. Kang, C. Lee, D. Kim, Hull form development and powering performance characteristics for a 2500 ton class Trimaran, Practical Design of ships and Other Floating Structures, (2001)

4. E. Jahanbakhsh, R. Panahi and M.S. Seif, Numerical Simulation of Three-Dimensional Interfacial Flows, International Journal of Numerical Methods for Heat \& Fluid Flow, Vol. 17, Issue 4, (2007)

5. H. Miyata, T. Sato and N. Babo, Difference solution of a viscous flow with free-surface wave about an advancing ship, J. Comput. Phys., Vol.72, p.393-421, (1987)

6. C. Hochbaum, A finite volume method for turbulent ship flows, Ship Technology Research Schiffstechnik, Hamburg, Germany, (1994)

7. B. Alessandrini and G. Delhommeau, Simulation of threedimensional unsteady viscous free surface flow around a ship model, Int. J. of Numerical Math. Fluids, Vol.19, p.321-342, (1994)

8. T. Kinoshita, H. Kagemoto and M. Fujino, A CFD application to wave-induced floating-body dynamics, 7th Int. Conference on Numerical Ship Hydrodynamics, Nantes, France, (1999)

9. R. Miyake, T. Kinoshita and H. Kagemoto, Ship Motions and loads in large waves, $23^{\text {rd }}$ ONR Symp. On Naval Hydrodynamics, Val de Reuil, France, (2000)

10.R. Azcueta, Computation of turbulent free surface flow around ships and floating bodies, PhD Thesis, Teschnichen Universitat Hamburg-Harburg, (2001) 
11.M. Vogt and C. Hochbaum, Numerical simulation of ship motions in head waves with a RANSE method, HSVA Report 1649, Hamburg, Germany, (2002)

12.Y. Xing-Kaeding, Unified approach to ship seakeeping and maneuvering by a RANSE method, $\mathrm{PhD}$ Thesis, Teschnichen Universitat Hamburg-Harburg, (2004)

13.E. Jahanbakhsh, R. Panahi and M.S. Seif, Ship dynamic simulation, based on a three-dimensional viscous free surface flow solver, $9^{\text {th }}$ Numerical Towing Tank Symposium (NuTTs'06), Le Croisic, (2006)

14.J.C. Park, H. Miyata, Numerical simulation of fully-nonlinear wave motions around arctic and offshore structures, J. Society of Naval Architects Japan, 2001, Vol.189, p. 13-19

15.R. Panahi, E. Jahanbakhsh and M.S. Seif, Development of a VoF-fractional step solver for floating body motion simulation, Applied Ocean Research, Volume 28, Issue 3, (2006)
16.C. Bertorello, D. Bruzzone, P. Cassella and I. Zotti, Trimaran model test results and comparison different high speed craft, Practical Design of Ships and Floating Structures, (2001)

\section{CONTACT WITH THE AUTHOR}

M.R. Javanmardi, E. Jahanbakhsh, M.S. Seif, H.Sayyaadi

Mechanical Engineering Department, Sharif University of Technology Tehran, IRAN

e-mail : seif@sharif.edu

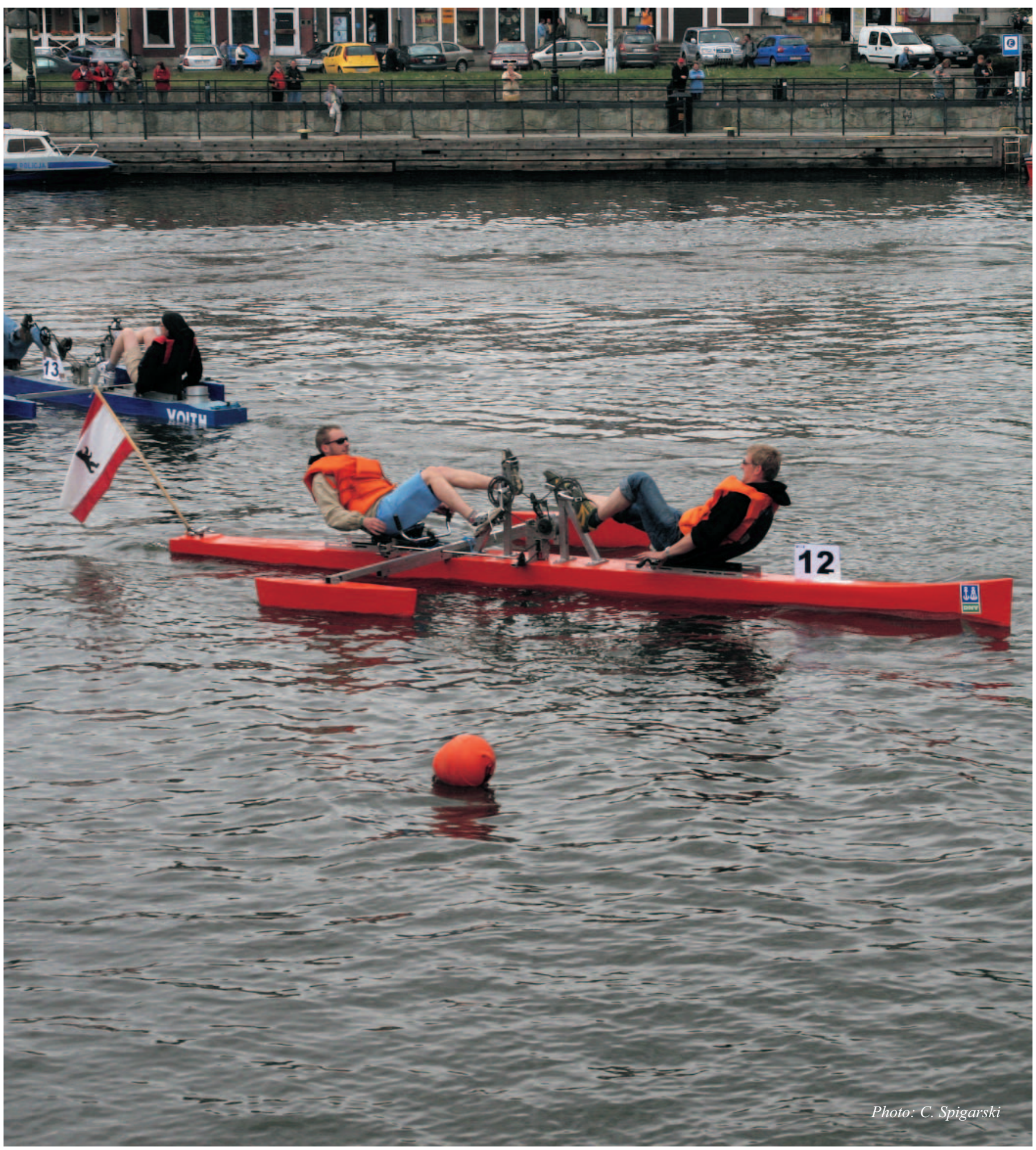

